\title{
Chronic Granulomatous Inflammation of the Breast as a First Clinical Manifestation of Primary Sarcoidosis
}

\author{
Petra Valković Zujića Damir Grebićb $^{b}$ Lara Valenčićc \\ a Department of Radiology, Clinical Hospital Center Rijeka, School of Medicine, University of Rijeka, Rijeka, Croatia; \\ ${ }^{b}$ Department of Surgery, Clinical Hospital Center Rijeka, School of Medicine, University of Rijeka, Rijeka, Croatia; \\ c Student of General Medicine, Medical Faculty of Rijeka, School of Medicine, University of Rijeka, Rijeka, Croatia
}

\section{Established Facts}

- Primary sarcoidosis of the breast is very rare. It occurs in less than $1 \%$ of cases and it can be confused with malignancy.

\section{Novel Insights}

- When changes in the breast are chronic and are presented as indeterminate masses it is mandatory to biopsy the breast to obtain the correct diagnosis and start proper treatment. In our case with primary breast sarcoidosis corticosteroid therapy was started early, which led to excellent improvement and remission of the disease.

\section{Keywords}

Breast sarcoidosis - Core needle biopsy .

Granulomatous disease

\section{Summary}

Background: Sarcoidosis is an idiopathic multisystemic disease that affects young to middle aged adults, with higher incidence in women. Although it may involve the breast parenchyma, primary sarcoidosis of the breast is very rare. It occurs in less than $1 \%$ of cases. In a differential diagnosis it may potentially be considered a malignancy. Case Report: We report a case in which breast sarcoidosis was the first clinical manifestation of systemic disease in a 54-year-old woman who presented with wide erythematous skin changes associated with palpable induration. Considering the fact that physical examination and the results of mammography, ultrasound and magnetic resonance imaging were inconclusive and unable to rule out malignancy, biopsy was performed. Pathohistological diagnosis showed a non-necrotizing granulomatous inflammation without elements of breast cancer. Sarcoidosis was confirmed with elevated level of angiotensin-converting enzyme in the sera and characteristic chest multislice computed tomography findings. The bronchoalveolar lavage was infiltrated with lymphocytes. Conclusion: Breast sarcoidosis has diverse and nonspecific imaging characteristics. Carcinoma must always be excluded by core needle biopsy. Achieving correct diagnosis is mandatory so that adequate corticosteroid therapy can be applied as early as possible. A multidisciplinary approach is of utmost importance in the diagnostic workup.

\section{KARGER \\ Fax +497614520714

\section{(c) 2015 S. Karger GmbH, Freiburg}

$1661-3791 / 15 / 0101-0051 \$ 39.50 / 0$ 
Fig. 1. Skin changes of the left breast between the upper quadrants partially covered with flakes.

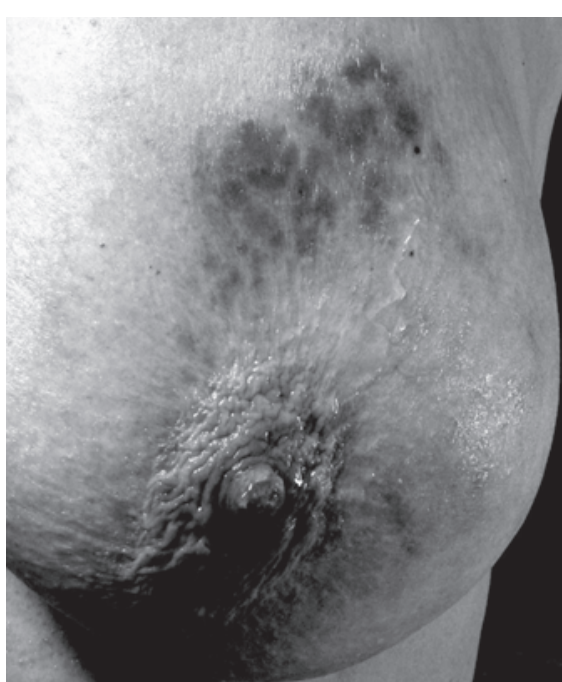

\section{Introduction}

Sarcoidosis is an idiopathic multisystemic disorder that affects young to middle aged adults, with a higher incidence in women. A typical pathohistological finding is noncaseating granuloma, which is characteristic for chronic granulomatous disease. Sarcoidosis mostly involves intrathoracic structures, affecting mediastinal lymph nodes and the lungs in $90 \%$ of cases [1-3]. The diagnosis is based on typical radiological manifestations supported by histological evidence, in particular thoracic radiograms, associated with clinical examination and an elevated serum angiotensin-converting enzyme (ACE) level, a pathognomonic feature of sarcoidosis. Exclusion of other granulomatous diseases is necessary. Sarcoidosis of the breast is very rare. It occurs in less than $1 \%$ of cases and in differential diagnosis may be potentially considered as a malignancy [4-6].

We report a sporadic case of a patient with granulomatous inflammation of the breast as a first clinical manifestation of primary sarcoidosis.

\section{Case Report}

A 54-year-old woman presented with extensive erythematous skin changes of the left breast associated with palpable induration and a history of occasional subfebrile temperature over the previous 5 months. Clinical examination and inspection confirmed livid, erythematous skin of the left breast between the upper quadrants, which showed brown pigmentation and was partially covered with flakes (fig. 1). On palpation, it presented as a solid induration of approximately $6 \mathrm{~cm}$ in diameter. Ipsilateral axillary lymph nodes were enlarged and indurated.

Ultrasonography of the left breast was performed utilizing a 15-MHz transducer Logiq E9 (Ge Medical System, Inc., Milwaukee, WI, USA). The entire breast tissue, including the subcutaneous area, was hyperechoic with thickened skin. Axillary lymph nodes were verified as reactive, with dimensions up to $22 \mathrm{~mm}$ (fig. 2). Furthermore, bilateral mammogram, classified as BI-RADS 0 (BI-RADS; Breast Imaging and Reporting Data System), showed a parenchymal blurring that was fused with the pectoral muscle. T2-weighted magnetic resonance imaging (MRI) with fat suppression revealed extensive inflammatory

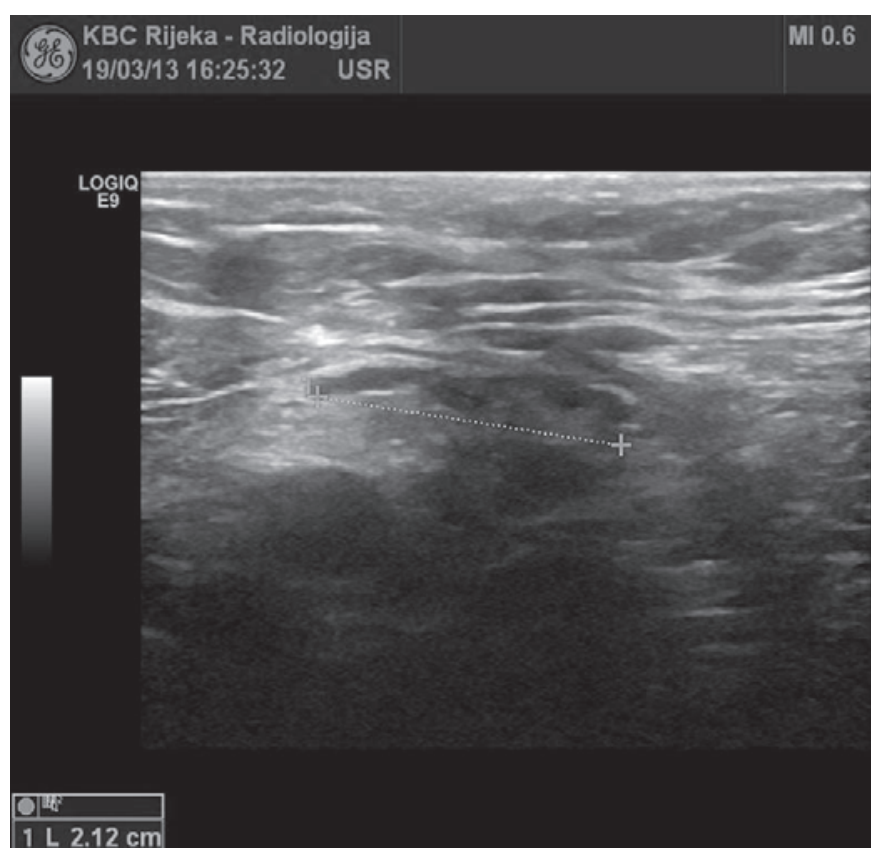

Fig. 2. Ultrasound image of reactive axillary lymph node.

Fig. 3. Contrast-enhanced T1-weighted magnetic resonance image shows irregular enhancing areas of the left breast and ipsilateral pectoral muscle involvement.

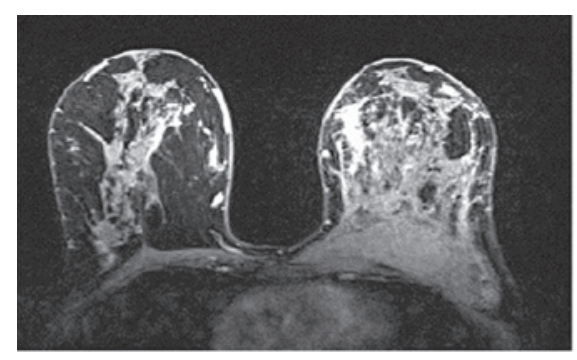

changes and lymphangitis of the left breast with an infiltration of the left pectoral muscle and enlarged ipsilateral axillary lymph nodes. Dynamic contrast-enhanced T1-weighted MRI showed irregular enhancing areas of the left breast with ipsilateral pectoral muscle involvement (fig. 3). Since inflammatory breast cancer could not be excluded with the obtained results, core needle biopsy (CNB) was performed. Pathohistological diagnosis revealed a non-necrotizing granulomatous inflammation that was consistent with sarcoidosis but not with inflammatory breast cancer. Fine-needle aspiration (FNA) of the left axillary lymph node showed a reactive lymph node indicative of reactive lymphadenitis. Fungal infection was excluded.

Based on the histological CNB diagnosis and the chronic course of the disease, which were suggestive of sarcoidosis, multislice computed tomography (MSCT) of the chest was performed, revealing characteristic multiple spiculated subpleural and perivascular nodules (fig. 4). Hyperdense focal lesions infiltrated the left breast fat tissue. Thickening of the breast cutis and subcutis with edema of the left pectoral muscle were detected. The left axillary lymph nodes were reactive. All these findings suggested the diagnosis of sarcoidosis.

Confirmation of the clinical-radiological findings and histological evidence suggestive of sarcoidosis was obtained using other significant and pathognomonic tests. The level of ACE in the sera was significantly elevated (90.1 U/1, normal range 20-70); the sera calcium concentration was at the upper level (2.53 mmol/l, normal range 2.14-2.53); the anti-nuclear antibody (ANA) test was negative; and bronchoalveolar lavage was infiltrated with lymphocytes (25\%).

Once the diagnosis was confirmed the patient was treated with a standarddose corticosteroid therapy $(0.5-1 \mathrm{mg} / \mathrm{kg} / \mathrm{day})$, with a gradual reduction in dosage. Remission of the disease was achieved and confirmed by a control breast MRI revealing no signs of relapse, and 2-year follow-up results. 
Fig. 4. On multislice computed tomography (MSCT), thin coronal maximum intensity projection (MIP) sections of the chest show multiple spiculated subpleural and perivascular nodules characteristic for pulmonary sarcoidosis.

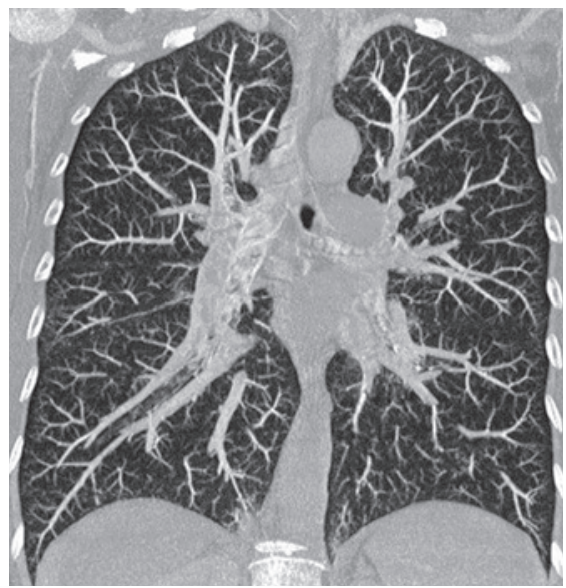

\section{Discussion}

We present a very rare clinical manifestation of primary sarcoidosis in the breast parenchyma. Sarcoidosis is a systemic chronic granulomatous disease that predominantly affects the intrathoracic organs. Only a few cases with a primary presentation of this disease in the breast tissue have been reported [7]. Sarcoidosis must be included in differential diagnosis of breast malignancy. In our case, the MRI findings suggested possible diffuse malignant infiltrations within an inflammatory carcinoma caused by lymphangitis of the left breast, with infiltration of breast tissue and left pectoral muscle. Ultrasonography and mammography also revealed an indeterminate, diffuse mass. Only a few case reports of breast sarcoidosis that were considered as possible breast cancer have been published [4, 7]. Although not specific, some reported cases suggested MRI as a method of choice for the diagnosis of breast sarcoidosis [8]. In our case, MRI could not exclude inflammatory breast cancer but CNB confirmed sarcoidosis. As biopsy is a specific method for this diagnosis, pathohistological verification of breast tissue should always be made. CNB suggested chronic granulomatous inflammation and FNA showed reactive left axillary lymph nodes with no suspicious malignant cells. As a result, both examinations excluded the possibility of malignant lesions. The importance of breast biopsy has already been stressed [9]. All their group of 629 women with sarcoidosis underwent breast biopsy, and the granulomatous changes of the breast were confirmed in
10 cases [9]. Although not a significant number of cases, it indicates the importance of breast biopsy when suspicious lesions are present.

Our diagnosis was also verified by the MSCT findings of characteristic multiple spiculated nodules with a subpleural and perivascular distribution in the middle lung zones, and thickened breast tissue with edema of the left pectoral muscle associated with enlarged and reactive left axillary lymph nodes. In our patient, all findings suggested sarcoidosis with lung involvement. This could be expected as the lungs are involved in $90 \%$ of cases with this disease [1-3]. The assessment of serum ACE activity, another specific test essential in diagnostics of primary sarcoidosis, also confirmed our findings.

In the differential diagnosis we excluded collagenous disorders by performing an ANA test. This test included antibodies against dsDNA, nRMP/SM, SS-A, SS-B, Scl-70, Jo-1, histones, ribosomal $\mathrm{P}$-proteins and centromere. All yielded negative results. Furthermore, other chronic granulomatous diseases such as idiopathic granulomatous mastitis, fungal infections and foreign body reactions had to be excluded $[7,10]$. Idiopathic granulomatous mastitis is characteristic for the postpartum period, and it is characterized with panlobulitis associated with microabscesses, ductal damage and ductal inflammation [10]. No microabscesses and ductal damage were present in our CNB findings. Fungal infection was excluded by negative microscopic findings. Biopsy showed no foreign body reaction.

In conclusion, breast sarcoidosis is a rare presentation of this systemic disease, especially as a first clinical manifestation. When changes in the breasts are chronic and present as indeterminate masses, biopsy is required for pathohistological verification and accurate diagnosis. CNB in association with high serum levels of ACE confirmed our diagnosis of primary breast sarcoidosis. Because of the prompt diagnosis, early corticosteroid therapy could be started. Our patient showed improvement and remission of the disease with excellent 2-year follow-up.

\section{Disclosure Statement}

The authors declare that they have no financial or nonfinancial relationships to disclose or any sponsorship or funding arrangements relating to our research.

\section{References}

1 Aladesanmi OA: Sarcoidosis: An update for the primary care physician. MedGenMed 2004;6:7.

2 Prabhakar HB, Rabinowitz BC, Gibbons FK, et al.: Imaging features of sarcoidosis on MDCT, FDG PET, and PET/CT. AJR Am J Roentgenol 2008;190:1-6.

3 Tremblay A, Stather DR, Maceachern P, et al.: A randomized controlled trail of standard vs endobronchial ultrasonography-guided transbronchial needle aspiration in patients with suspected sarcoidosis. Chest 2009; 136:340-346.
4 Isley LM, Cluver AR, Leddy RY, Baker MK: Primary sarcoid of the breast with incidental malignancy. J Clin imaging Sci 2012;2:46.

5 Fiorucci F, Conti V, Lucantoni G, et al.: Sarcoidosis of the breast: A rare case report and a review. Eur Rev Med Pharmacol Sci 2006;10:47-50.

6 Donaldson BA, Polynice A, Oluwole S: Sarcoidosis of the breast: Case report and chart review. Am Surg 1995; 61:778-780.
7 Gisvold JJ, Crotty TB, Johnson RE: Sarcoidosis presenting as spiculated breast masses. Mayo Clin Proc 2000; 75:293-295.

8 Krause A, Gerber B, Rohde E, Kuchenmeister I: Sarcoid tumor of the breast (in German). Zentralbl Gynakol 1996;118:299-302.

9 Mona EK, Pascala C, Charleya H, et al.: Quiz case. Breast sarcoidosis presenting as a metastatic breast cancer. Eur J Radiol 2005;54:2-5.

10 Banik S, Bishop PW, Ormerod LP, O’Brien TE: Sarcoidosis of the breast. J Clin Pathol 1986;39:446-448. 